\title{
Hyperostosis Frontalis Interna Requiring Craniotomy for Intracranial Hypertension
}

\author{
Cameron Elliott, Edward Johnson, Michael Chow
}

Can J Neurol Sci. 2014; 41: 109-111

Hyperostosis frontalis interna (HFI) is a characteristically benign metabolic craniopathy involving focal lamellar bone deposition along the inner table of the frontal bones. Although some cases of HFI may be symptomatic with intrusive bone growth causing cognitive slowing, seizures or mood disturbance, rarely will HFI present in a dramatic manner. Here, we report a case of HFI causing marked intracranial hypertension, tonsilar herniation and bilateral posterior cerebral artery (PCA) ischemia treated successfully with bi-hemispheric decompressive craniotomy and cranioplasty.

\section{Case Presentation}

A 30-year-old woman with a history of intermittent frontal headaches and syncopal attacks thought to be secondary to basilar migraines presented to the emergency department following a prolonged loss of consciousness accompanied by emesis requiring endotracheal intubation. Examination revealed a Glasgow Coma Scale score of 8, a right oculomotor nerve palsy and bilateral, 5-mm, non-reactive pupils.

\section{Imaging and Investigations}

A computed tomogram (CT) scan of the head revealed diffuse calvarial thickening with effacement of basal cisterns and cortical sulci (Figure 1). Magnetic resonance imaging (MRI) demonstrated fourth ventricle compression and early tonsilar herniation accompanied by brain stem and bilateral small posterior cerebral artery territory infarcts (Figure 1). A MRI obtained several years earlier did not show evidence of a Chiari malformation. Catheter angiography revealed no unexpected abnormality, specifically no evidence of vertebrobasilar stenosis. Dual intraparenchymal and external ventricular drain pressure monitoring revealed intracranial pressures (ICP) of $18-62$ $\mathrm{mmHg}$, with occasional spikes of high-pressure waves lasting up to ten minutes. There were no biochemical abnormalities with alkaline phosphatase, calcium, phosphate, parathyroid hormone and growth hormone all within normal limits.

\section{Treatment}

In the absence of any other etiology, the intracranialhypertension was thought to be secondary to bi-hemispheric calvarial thickening and the patient underwent a bilateral osteoplastic decompressive frontotempoparietal craniotomy and cranioplasty. Minimal dural adhesions were encountered. An expansile duraplasty was not performed. Inner table bone overgrowth was drilled away to approximately one-half its original thickness and bone flaps were reattached in the typical

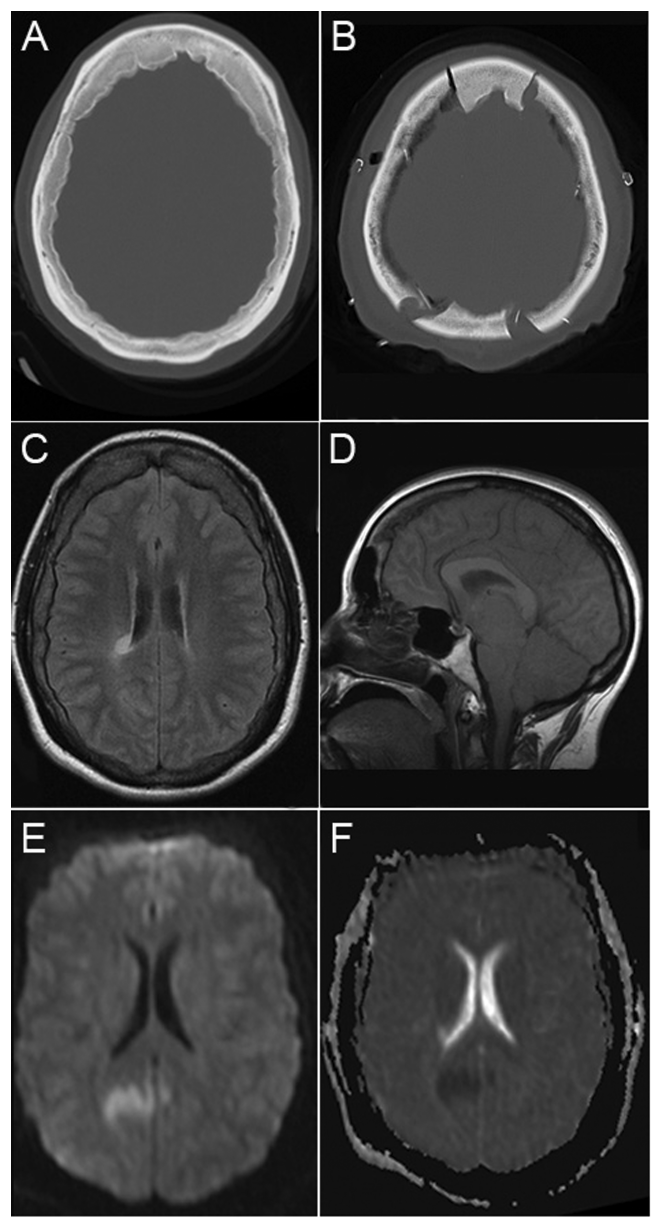

Figure 1: A: Axial CT demonstrating nodular bony thickening focused on both frontal bones extending posteriorly with relative sparing of midline anteriorly. B: Axial CT following bi-hemispheric craniotomy and cranioplasty revealing the extent of bony decompression achieved. $C$ : Axial T1-weight MR image revealing cortical sulcul effacement. D: Sagittal T1-weighted MR image show crowding of the posterior fossa structures including effacement of the fourth ventricle and tonsilar herniation. E/F: Axial B1000 diffusion-weighted imaging and ADC map revealing small region of acute posterior cerebral artery (PCA) distribution infarct.

From the Divisions of Neurosurgery (CE, MC) and Anatomical Pathology (EJ), University of Alberta, Edmonton, Alberta, Canada.

Received August 19, 2013. Final Revisions Submitted August 23, 2013. Correspondence to: Cameron Elliott, Walter Mackenzie Health Sciences Centre, University of Alberta, Department of Neurosurgery, 8440 - 112 Street, Edmonton, Alberta, T6G 2B7, Canada. Email:celliott@ualberta.ca. 
fashion. A full thickness biopsy of bone was obtained. An intraparenchymal pressure monitor was re-inserted.

\section{Histological Examination}

The $1.5 \mathrm{~cm}$ thick biopsy of bone, following decalcification, shows diffuse hyperostosis involving the outer table, trabeculae of the diploe, and resected portion of the inner table by formation of mature lamellar bone (Figure 2). Because of this hyperostosis, the intramedullary spaces in the diploe are narrowed and in the portion of inner table effaced. There are no features of recent bone formation (deposition of cement lines, osteoblastic activity, and osteoclastic resorption) dysplasia, or neoplasia.

\section{Postoperative Course}

Following decompression, ICP returned to normal $(2-15$ $\mathrm{mmHg}$ ) and the patient was extubated uneventfully. The patient's headaches disappeared and she remained free from further syncopal attacks. Following a brief inpatient stay on a brain injury rehabilitation unit for ongoing issues with diplopia and cognitive dysfunction she was discharged home. When last seen in follow-up four years after surgery, she was headache free, independent and has been able to return to driving with the delayed resolution of diplopia. The CT imaging has demonstrated no re-growth of the inner table of the skull.

\section{Discussion}

Hyperostosis frontalis interna is characterized by insidious, bilateral, nodular expansion of well-organized, cancellous bone largely limited to the inner table of the frontal bones. Bone deposition typically spares the midline falx cerebri, superior sagittal sinus as well as the middle meningeal artery, posterolaterally. ${ }^{1,2}$ Originally described, in 1719 , as part of a syndrome of obesity and virility by Morgagni and Santorini, HFI is now thought to represent an independent clinical entity of uncertain etiology. ${ }^{1}$ Prevalence in the general population ranges from 5 $12 \%$, however, it may occur in up to $60 \%$ of post-menopausal, elderly women.

Hyperostosis frontalis interna is typically asymptomatic, but may present with headaches, cognitive impairment, mood disturbance or seizures. ${ }^{3}$ Probable diagnosis is based on imaging characteristics, while definitive diagnosis requires a surgical specimen which is reserved for clearly symptomatic cases in which no alternative, less invasive treatment option is feasible.

The differential diagnosis of cranial hyperostosis includes diffuse processes such as Paget's disease, acromegaly, osteopetrosis, chronic cerebrospinal fluid-diversion (e.g. ventriculoperitoneal shunt), long-term antiepileptic agent use and hyper-parathyroidism as well as focal processes such as fibrous dysplasia, meningioma, ossifying fibroma and HFI. Monostotic fibrous dysplasia was a diagnostic consideration, as it commonly involves the frontal bone or maxilla, however it typically results in displaced bowing of the outer table, with cosmetic deformity and histologically is characterized by replacement of normal lamellar bone by fibro-osseous tissue containing woven bone. ${ }^{2}$ Given the focal location of the bone pathology in this patient, lack of biochemical abnormalities and histologic features as described, HFI is the most likely diagnosis.

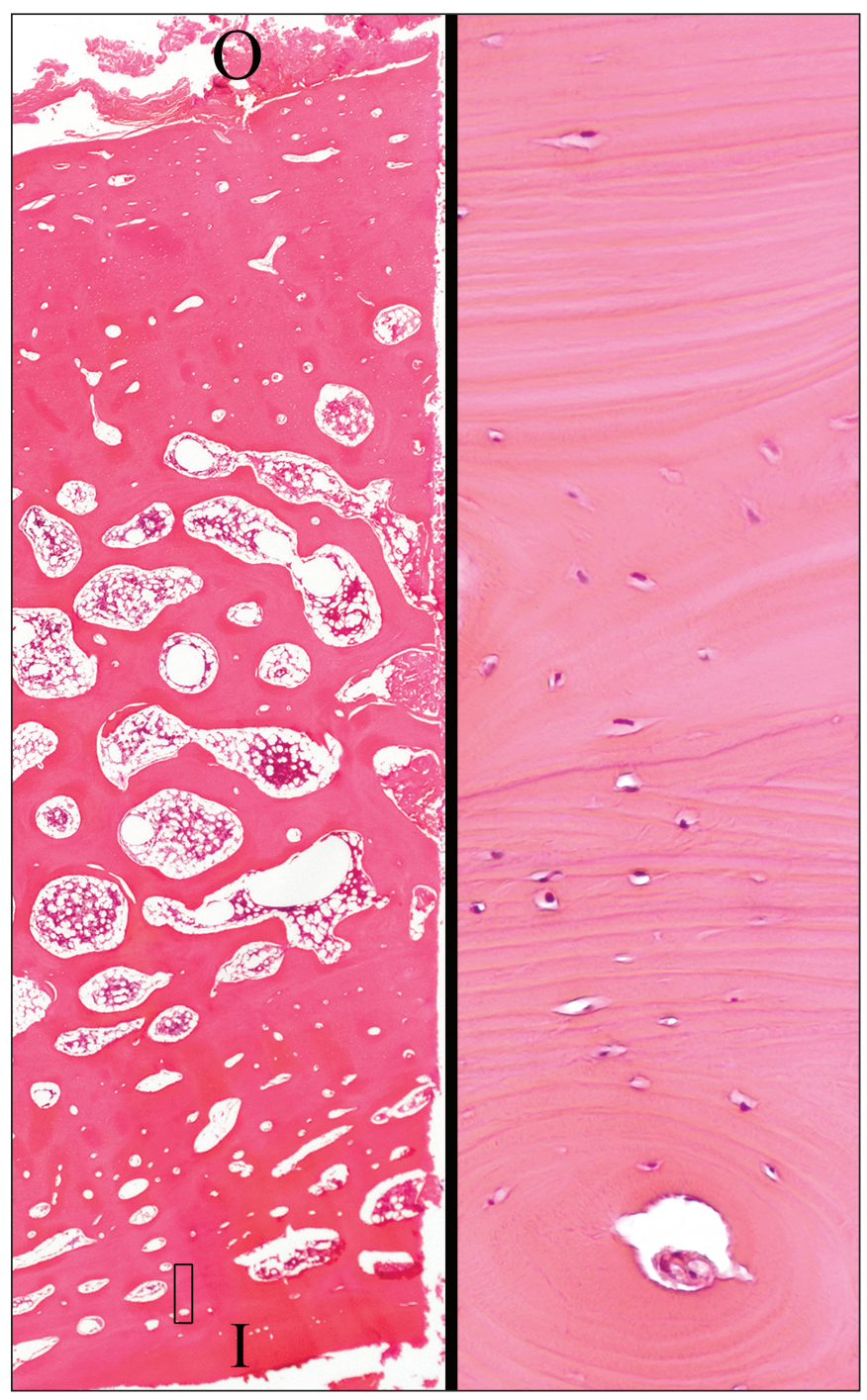

Figure 2: Biopsy of calvarium showing, left, full thickness of bone with diffuse hyperostosis involving the outer table $(O)$, inner table $(I)$, and diploe. High magnification of area in box, right, demonstrates mature lamellar bone with prominent cement lines and osteocytes. Stain: $H \& E$; magnification left $x 18$, right $\times 200$.

Hyperostosis frontalis interna, although a relatively common metabolic craniopathy, especially among at risk individuals (elderly, female, post-menopausal), is rarely so clearly and dramatically symptomatic. Extensive calvarial involvement with HFI and presentation of HFI in patients as young as 19-years-old have been previously reported, although in two different cases. ${ }^{2,4}$ The clinical history is congruent with long-standing vertebrobasilar insufficiency that eventually resulted in posterior fossa strokes. The combination of this patient's relatively young age and lack of cerebral atrophy with moderately extensive hyperostosis set the stage for her described deterioration. Our management of this case via decompressive cranial vault remodeling has previously been employed in a more diffuse cranial hyperostosis secondary to progressive diaphysial 
dysplasia (Camurati-Engelmann's disease) similarly causing elevated $\mathrm{ICP}^{5}$ and in the setting of Proteus syndrome. ${ }^{6}$ This case represents the first reported incidence of its use for HFI.

\section{SUMMARY}

We describe an atypical case of HFI associated with marked intracranial hypertension and posterior fossa ischemia successfully treated with bi-hemispheric cranial vault remodeling.

\section{REFERENCES}

1. Moore S. Calvarial hyperostosis and accompanying symptomcomplex. Arch Neurol Psychiat. 1936;35:975.

2. Chen OI, Murthy AS. Benign hyperostotic mass of the frontal bone. J Craniofac Surg. 2012;23(1):e27-30.

3. Hasegawa T, Ito H, Yamamoto S. Unilateral hyperostosis frontalis interna: case report. J Neurosurg. 1983;59:710-13.

4. Chaljub G, Johnson RF III, Johnson RF Jr, et al. Unusually exuberant hyperostosis frontalis interna: MRI. Neuroradiology. 1999;414:44-5.

5. Mocco J, Komotar RJ, Zacharia BE, Feldstein NA, Bruce JN. Aggressive cranial vault decompression for cranial hyperostosis: technical case report of two cases. Oper Neurosur. 2005;57(ONS Suppl 1):212.

6. Dandine JB, James S, Van Garsse A, Born JD. Intracranial hypertension in Proteus syndrome. Neurochirurgie. 2007;53(5): 339-42. 